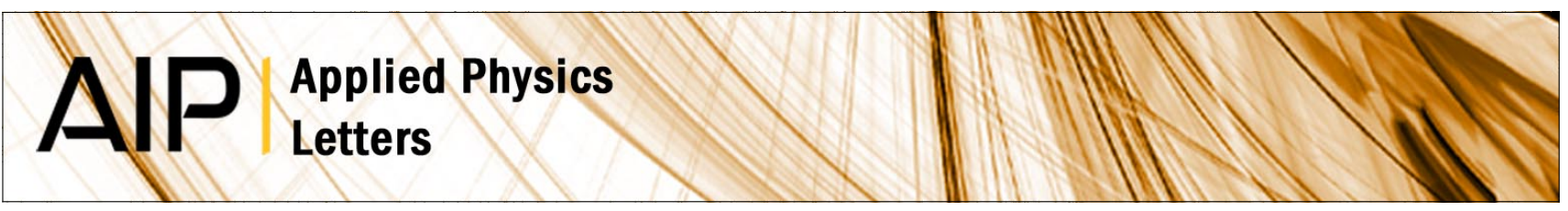

\title{
Analytic perturbation solution to the capacitance system of a hyberboloidal tip and a rough surface
}

Yongxing Shen, David M. Barnett, and Peter M. Pinsky

Citation: Appl. Phys. Lett. 92, 134105 (2008); doi: 10.1063/1.2906487

View online: http://dx.doi.org/10.1063/1.2906487

View Table of Contents: http://apl.aip.org/resource/1/APPLAB/v92/i13

Published by the American Institute of Physics.

\section{Related Articles}

Ambient effects on electric-field-induced local charge modification of TiO2

Appl. Phys. Lett. 100, 022901 (2012)

Control of selective adsorption behavior of Ti-binding ferritin on a SiO2 substrate by atomic-scale modulation of local surface charges

Appl. Phys. Lett. 99, 263701 (2011)

Resonant tunneling in $\mathrm{Si} / \mathrm{SiGe} / \mathrm{Si}$ structures with a single quantum well under surface passivation

J. Appl. Phys. 110, 123710 (2011)

On the true optical properties of zinc nitride

Appl. Phys. Lett. 99, 232112 (2011)

Structural dynamics of surfaces by ultrafast electron crystallography: Experimental and multiple scattering theory J. Chem. Phys. 135, 214201 (2011)

\section{Additional information on Appl. Phys. Lett.}

Journal Homepage: http://apl.aip.org/

Journal Information: http://apl.aip.org/about/about_the_journal

Top downloads: http://apl.aip.org/features/most_downloaded

Information for Authors: http://apl.aip.org/authors

\section{ADVERTISEMENT}

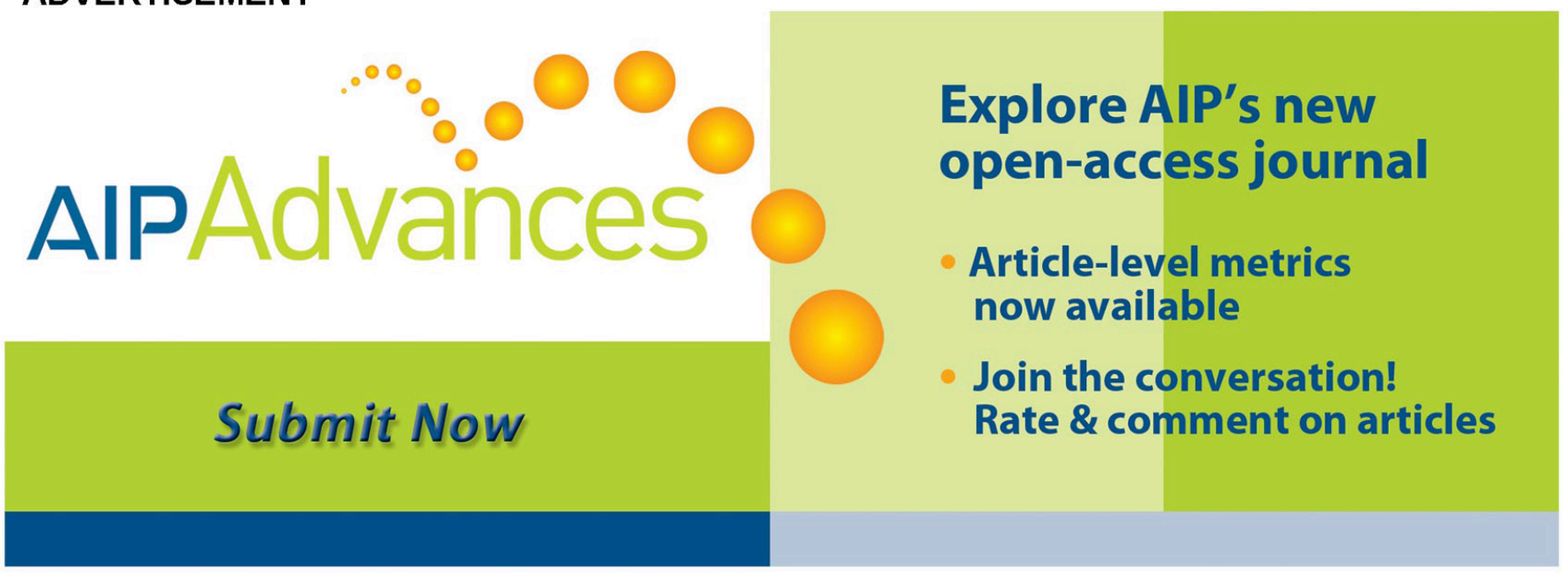




\title{
Analytic perturbation solution to the capacitance system of a hyberboloidal tip and a rough surface
}

\author{
Yongxing Shen, ${ }^{1, a)}$ David M. Barnett, ${ }^{1,2}$ and Peter M. Pinsky ${ }^{2}$ \\ ${ }^{1}$ Department of Materials Science and Engineering, Stanford University, Stanford, California 94305, USA \\ ${ }^{2}$ Department of Mechanical Engineering, Stanford University, Stanford, California 94305, USA
}

(Received 18 October 2007; accepted 17 March 2008; published online 4 April 2008)

\begin{abstract}
The capacitance system of a hyperboloidal tip and a rough surface is usually encountered in analyzing electrostatic force microscopy images. In this letter, a perturbation approach has been applied to solve for the electric potential of this system, in which the rough surface is treated as perturbation from a flat one. For the first-variation solution, the boundary value problem is represented in the prolate-spheroidal coordinate system and solved in terms of a generalized Fourier series involving conical functions. Based on this solution, the tip-surface Coulombic interaction can be computed. Sample calculations have been applied to sinusoidal surface profiles. (C) 2008 American Institute of Physics. [DOI: 10.1063/1.2906487]
\end{abstract}

Capacitance problems are one of the most studied types of boundary value problems and are applied not only in electrostatic and steady-current computations but also in steady heat conduction simulations. However, such problems involving one or two rough electrodes usually forbid exact analytic solutions, with the exception of some twodimensional problems for which conformal mappings may be used (see, e.g., Ref. 1). In general, although numerical techniques such as the finite element method are readily available and sufficiently accurate for most engineering applications, they might lack insights when used for analyzing the behaviors of a family of similar problems, e.g., capacitance systems with a continuously varying electrode spacing.

On the other hand, approximate analytic solutions to capacitance problems with a rough electrode may serve as alternative methodologies capable of investigating the dependence of interested parameters. Such solutions include the solution of Hurych ${ }^{2}$ to the capacitor of a dielectric thin film of nonuniform thickness, the perturbation approach of García-Valenzuela et $a l .^{3,4}$ to periodic plate capacitors, and the formula of Boyer et al. ${ }^{5}$ for the capacitance between a sphere and a rough plane.

In this letter, we have investigated the capacitance system of a sheet of a two-sheeted hyperboloid and a rough surface, which may find applications in analyzing images of electrostatic force microscopy (EFM). ${ }^{6}$ As a variant of atomic force microscopy, EFM measures the electrostatic interactions between a probe tip and materials such as semiconductors (see Ref. 7 and references therein) in order to help understand their local charge distributions.

We will first solve the capacitance system of a hyperboloid and a flat surface, and then treat the rough surface as perturbation from the flat surface by introducing a compensating boundary condition (BC) on the flat surface.

To accommodate the geometry, we introduce the prolate spheroidal coordinates $(\xi, \eta, \phi):^{8}$

$$
\rho=a \sqrt{\xi^{2}-1} \sqrt{1-\eta^{2}}, \quad \phi=\phi, \quad z=a \xi \eta,
$$

where $\rho, \phi$, and $z$ are cylindrical coordinates and $a>0$ is a constant. The domains of the prolate spheroidal coordinates

\footnotetext{
${ }^{a)}$ Electronic mail: shenyx@stanford.edu.
}

are $1 \leqslant \xi<+\infty,-1 \leqslant \eta \leqslant 1,0 \leqslant \phi \leqslant 2 \pi$. Note that the surfaces with constant $\xi(>1)$ represent a family of confocal prolate spheroids, whereas those with constant $\eta$ are confocal hyperboloids of two sheets (except for $\eta=0$ which represents a plane). The foci of these spheroids and hyperboloids are located on the $z$ axis with $z= \pm a$. The metrics of the prolate-spheroidal coordinates are

$$
\begin{gathered}
h_{\xi}=a \sqrt{\frac{\xi^{2}-\eta^{2}}{\xi^{2}-1}}, \quad h_{\eta}=a \sqrt{\frac{\xi^{2}-\eta^{2}}{1-\eta^{2}}}, \\
h_{\phi}=a \sqrt{\left(\xi^{2}-1\right)\left(1-\eta^{2}\right)} .
\end{gathered}
$$

In the reference capacitance problem of a tip and a flat surface, the two electrodes are modeled as surfaces with constant $\eta$ 's: $\eta=\eta_{1}\left(0<\eta_{1}<1\right)$ for the tip surface and $\eta=0$ for the flat surface. The probe-tip radius of curvature $R$, the half cone angle of the tip $\theta$, and the tip-sample separation $d$ are given by $a\left(1-\eta_{1}^{2}\right) / \eta_{1}, \cos ^{-1} \eta_{1}$, and $a \eta_{1}$, respectively, ${ }^{9}$ (see Fig. 1). Note that only two of these three quantities are in-

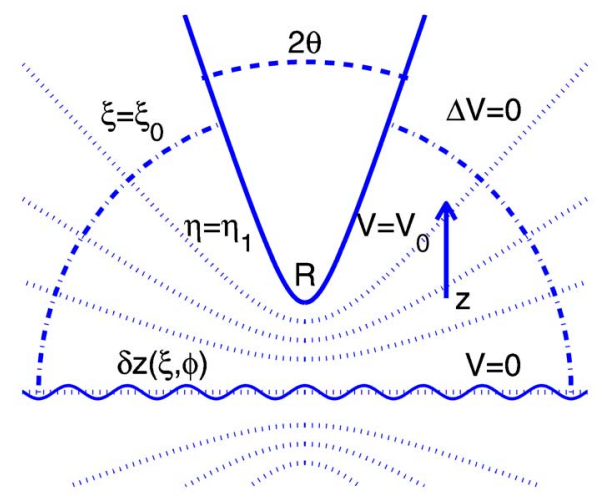

FIG. 1. (Color online) Schematic of exact and approximated electrostatic BVPs between a hyperboloidal tip and a surface with topography. The boundaries of the exact BVP are shown as solid curves. This BVP is approximated as perturbation from a reference solution $\bar{V}$, in which BCs are imposed on the tip $\eta=\eta_{1}$ and the flat surface $\eta=0$. The first-variation solution $\delta V$ is determined by imposing compensating BCs on $\eta=\eta_{1}$ and $\eta=0$. To ease the numerical implementation, an artificial BC $\partial(\delta V) / \partial \mathbf{n}=0$ is imposed on the prolate spheroid $\xi=\xi_{0}$ (dash-dotted curve). This prolate spheroid is determined such that it encloses the actual size of the tip (not drawn to scale in the figure). 
dependent in this model.

For quantities in the reference problem, we will use notations with overbars. Since there is no charge present in the region between surfaces $\eta=\eta_{1}$ and $\eta=0$, the potential of the reference problem $\bar{V}$ satisfies Laplace's equation $\Delta \bar{V}=0$. In the prolate spheroidal coordinates, the Laplacian operator $\Delta$ reads $^{8}$

$$
\begin{aligned}
\Delta= & \frac{1}{a\left(\xi^{2}-\eta^{2}\right)}\left\{\frac{\partial}{\partial \xi}\left[\left(\xi^{2}-1\right) \frac{\partial}{\partial \xi}\right]+\frac{\partial}{\partial \eta}\left[\left(1-\eta^{2}\right) \frac{\partial}{\partial \eta}\right]\right. \\
& \left.+\frac{\xi^{2}-\eta^{2}}{\left(\xi^{2}-1\right)\left(1-\eta^{2}\right)} \frac{\partial^{2}}{\partial \phi^{2}}\right\} .
\end{aligned}
$$

Without loss of generality, we set the BCs for this reference problem to be $\bar{V}\left(\xi, \eta_{1}, \phi\right)=V_{0}$ and $\bar{V}(\xi, 0, \phi)=0$. The solution to this problem is $\bar{V}(\xi, \eta, \phi)=Q_{0}(\eta) V_{0} / Q_{0}\left(\eta_{1}\right)$, where $Q_{0}(\eta) \equiv(1 / 2) \ln [(1+\eta) /(1-\eta)]$ is the Legendre function of the second kind of degree 0 .

The tip-surface electrostatic force can be written as

$$
\bar{F}=\int_{S_{\text {tip }}} \frac{\bar{\sigma}^{2} n_{z}}{2 \epsilon_{0}} d S,
$$

where $\epsilon_{0}=8.8542 \times 10^{-12} \mathrm{~F} / \mathrm{m}$ is the vacuum permittivity, $\bar{\sigma}=-\epsilon_{0}[\partial \bar{V} / \partial \mathbf{n}]_{\eta=\eta_{1}}=\epsilon_{0}\left[h_{\eta}^{-1} \partial \bar{V} / \partial \eta\right]_{\eta=\eta_{1}}$ is the surface charge density, and $\mathbf{n}$ is the unit outward normal of the tip surface whose $z$ component is $n_{z}=-\xi\left[\left(1-\eta_{1}^{2}\right) /\left(\xi^{2}-\eta_{1}^{2}\right)\right]^{1 / 2}$. The area element $d S$ is given by $h_{\xi} h_{\phi} d \xi d \phi=a^{2}\left[\left(\xi^{2}-\eta_{1}^{2}\right)(1\right.$ $\left.\left.-\eta_{1}^{2}\right)\right]^{1 / 2} d \xi d \phi$. The integral in Eq. (4) diverges if one integrates over the entire surface $\eta=\eta_{1}(\xi$ from 1 to $\infty)$. To render a finite value, one must only integrate over a finite portion of this surface corresponding to the actual size of the hyperboloid: $1 \leqslant \xi \leqslant \xi_{0} \equiv 1+L / d$, where $L$ is the length of the entire probe in the axial direction. By doing so, one has $\bar{F}=-\left[\pi \epsilon_{0} V_{0}^{2} / 2 Q_{0}\left(\eta_{1}\right)^{2}\right] \ln \left[\left(\xi_{0}^{2}-\eta_{1}^{2}\right) /\left(1-\eta_{1}^{2}\right)\right]$.

Now we consider the problem with the flat surface $\eta=0$ replaced by a rough one $z=\delta z(\xi, \phi)$ (see Fig. 1), or, invoking (1), $\eta=\eta_{s}(\xi, \phi) \equiv(a \xi)^{-1} \delta z(\xi, \phi)$. The new (exact) boundary value problem (BVP) has BCs $V=V_{0}$ on $\eta=\eta_{1}$ and $V=0$ on $\eta=\eta_{s}(\xi, \phi)$, which does not allow an exact analytic solution for a generic surface profile $\delta z$.

For small roughness $(\|\delta z\| \ll a)$, however, we can treat the rough surface as perturbation from the flat surface. Here $\|\cdot\|$ denotes a certain measure for a function. To do so, we first extend the potential $\bar{V}(\xi, \eta, \phi)=Q_{0}(\eta) V_{0} / Q_{0}\left(\eta_{1}\right)$, to the region $-\eta_{1} \leqslant \eta \leqslant \eta_{1}$. Also note that since $\bar{V}$ vanishes on $\eta=0$ and its local gradient is perpendicular to that surface, the $\bar{V}$ values on the rough surface $\eta=\eta_{s}(\xi, \phi)$ can be approximated using a Taylor series expansion about the flat surface $\eta=0$,

$$
\bar{V}\left[\xi, \eta_{s}(\xi, \phi), \phi\right]=\frac{\partial \bar{V}}{\partial \eta}(\xi, 0, \phi) \eta_{s}(\xi, \phi)+O\left(\|\delta z\|^{2}\right) .
$$

Hence, if we could find a harmonic potential $\delta V$ with $\mathrm{BCs} \quad \delta V=0 \quad$ on $\quad \eta=\eta_{1} \quad$ and $\delta V=V_{s}(\xi, \phi)$ $\equiv-[\partial \bar{V} / \partial \eta]_{\eta=0} \eta_{s}(\xi, \phi)$ on $\eta=\eta_{s}(\xi, \phi)$, then $\bar{V}+\delta V$ would be a first-order approximation to the solution of the potential between the tip and the rough sample. To take advantage of the geometry, we will, however, impose the BC $\delta V$ $=V_{s}(\xi, \phi)$ on the flat surface $\eta=0$ instead. Since the value of this $\mathrm{BC}$ is already on the order of $\|\delta z\|$, this further approxi- mation only results in a second-order deviation, provided $\|\delta z\| \ll a$ and $\|\nabla \delta z\| \ll 1$.

Hence, we will solve for the first-variation correction $\delta V$ with Dirichlet BCs $\delta V=0$ on $\eta=\eta_{1}$ and $\delta V=V_{s}(\xi, \phi)$ on $\eta=0$ (see Fig. 1).

Using the method of separation of variables, we write $\delta V(\xi, \eta, \phi)=\Xi(\xi) H(\eta) \Phi(\phi)$. Applying Eq. (3) to $\delta V$ and equating the resulted field to zero, we have

$$
\begin{aligned}
& \frac{d}{d \xi}\left[\left(1-\xi^{2}\right) \frac{d \Xi}{d \xi}\right]+\left[\nu(\nu+1)-\frac{m^{2}}{1-\xi^{2}}\right] \Xi=0, \\
& \frac{d}{d \eta}\left[\left(1-\eta^{2}\right) \frac{d H}{d \eta}\right]+\left[\nu(\nu+1)-\frac{m^{2}}{1-\eta^{2}}\right] H=0, \\
& \frac{d^{2} \Phi}{d \phi^{2}}+m^{2} \phi=0,
\end{aligned}
$$

where $m=0,1,2, \cdots$ (which follows from the periodicity in $\phi)$ and $\nu$ is in general a complex number. Equations (6) and (7) are associated Legendre differential equations of degree $\nu$ and order $m$. Without loss of generality, a typical normal solution is $P_{\nu}^{m}(\xi) P_{\nu}^{m}( \pm \eta)_{\sin }^{\cos }(m \phi)$, where $P_{\nu}^{m}(\mu)$ is the associated Legendre function of the first kind. According to Snow ${ }^{10}$ a complete set is given by setting $\nu=-(1 / 2)+i p$ and letting $p$ assume real values from $-\infty$ to $\infty$. In this case, $K_{p}^{m}(\mu) \equiv P_{-(1 / 2)+i p}^{m}(\mu)$ is called a conical function. We can write $\delta V$ as a superposition of the normal solutions with (generalized) Fourier coefficients to be determined. These Fourier coefficients can be formally derived using the BCs and their mutual orthogonality (see Ref. 11). However, the subsequent numerical integration with respect to $p$ is challenging (see Ref. 9) due to the oscillating integrand.

To overcome this difficulty, we impose an artificial homogeneous Neumann BC on a prolate spheroid surface $\xi$ $=\xi_{0}$ (see Fig. 1) that encloses the actual size of the tip, and truncate the region exterior to this prolate spheroid. Now the domain is bounded by the prolate spheroid $\xi=\xi_{0}$, the probe surface $\eta=\eta_{1}$, and the flat surface $\eta=0$.

This additional BC does not alter the reference solution $\bar{V}$. For $\delta V$, it reduces to $\Xi^{\prime}\left(\xi_{0}\right)=0$ for Eq. (6), which leads to a Sturm-Liouville eigensystem for each $m$, allowing only discrete $p$ values such that $K_{p}^{m^{\prime}}\left(\xi_{0}\right)=0$. In an ascending order $p_{n}^{m}$, the $n$th root of $K_{p}^{m^{\prime}}\left(\xi_{0}\right)=0$ can be shown to be approximately $[n+(m+1) / 4)] \pi / \ln \left(2 \xi_{0}\right)$ for $\xi_{0} \gg 1$, which can serve to be initial values for root-finding routines. Denoting $T_{n}^{m}(\mu) \equiv K_{p_{n}^{m}}^{m}(\mu)$, we have the orthogonal condition

$$
\int_{1}^{\xi_{0}} T_{n}^{m}(\xi) T_{s}^{m}(\xi) d \xi=0, \quad \text { if } n \neq s .
$$

These orthogonal functions $\left\{T_{n}^{m}(\xi): n=1,2, \cdots\right\}$ constitute a complete set in $L_{2}\left(\left[1, \xi_{0}\right]\right)$ for each $m$ except when $m=0$, in which case we need an additional basis function for $\nu=0$ [i.e., $P_{0}(\xi)=1$ ]. This basis function corresponds to dependence on $\eta$ as $A_{0} P_{0}(\eta)+B_{0} Q_{0}(\eta)$, or simply $A_{0}$ $+B_{0} Q_{0}(\eta)$. Now $\delta V$ can be written as a double summation 


$$
\begin{aligned}
\delta V(\xi, \eta, \phi)= & A_{0}+B_{0} Q_{0}(\eta)+\sum_{m=0}^{\infty} \sum_{n=1}^{\infty} T_{n}^{m}(\xi) \\
& \times\left[A_{n}^{m} T_{n}^{m}(\eta) \cos (m \phi)+B_{n}^{m} T_{n}^{m}(-\eta) \cos (m \phi)\right. \\
& \left.+C_{n}^{m} T_{n}^{m}(\eta) \sin (m \phi)+D_{n}^{m} T_{n}^{m}(-\eta) \sin (m \phi)\right],
\end{aligned}
$$

where $A_{0}, B_{0}, A_{n}^{m}, B_{n}^{m}, C_{n}^{m}$, and $D_{n}^{m}$ are Fourier coefficients to be determined by matching $\operatorname{BCs} \delta V\left(\xi, \eta_{1}, \phi\right)=0$ and $\delta V(\xi, 0, \phi)=V_{s}(\xi, \phi)$ and invoking the orthogonal relation (9) and that between $\underset{\sin }{\cos }(m \phi), m=0,1,2 \cdots$.

With the first-order correction $\delta V$, one can obtain the correction for the surface charge density $\bar{\sigma}$ of the same order as $\delta \sigma(\xi, \phi)=\epsilon_{0}\left[h_{\eta}^{-1} \partial(\delta V) / \partial \eta\right]_{\eta=\eta_{1}}$. From Eq. (4), the firstorder correction for the force in the $z$ direction is given by $\delta F=\epsilon_{0}^{-1} \int_{S_{\text {tip }}} \bar{\sigma} \delta \sigma n_{z} d S$. By inspection, one deduces that only the axisymmetric part (terms with $m=0$ ) of $\delta \sigma$ contribute to $\delta F$. As a result, $\delta F$ only depends on the $\phi$-averaged topography $\delta \widetilde{z}(\xi) \equiv(1 / 2 \pi) \int_{0}^{2 \pi} \delta z(\xi, \phi) d \phi$.

As a sample application of this methodology, we set $V_{0}=1 \mathrm{~V}, \eta_{1}=0.950$, and $\xi_{0}=200$, which corresponds to $R=0.103 a, \theta=18.2^{\circ}$, and $d=0.950 a$ (see Fig. 1). In the reference solution, the tip-surface interaction $\bar{F}$ is given by Eq. (4) which is scale-independent and equals $-53.57 \mathrm{pN}$ (the minus sign meaning an attractive force). For numerically evaluating $\delta F$, we first use the built-in function "FindRoot" of the software WOLFRAM MATHEMATICA to solve for the roots $p_{n}^{0}, n=1,2, \cdots$. We find that the first $n_{\max }=10$ Fourier modes are sufficient for obtaining three significant digits. We then compute $\delta F$ as described in the previous paragraph and tabulate the results for constant and sinusoidal topographies in Table I. For example, if $a=50 \mathrm{~nm}$, the corresponding $d$ is $0.95 a=47.5 \mathrm{~nm}$. For a constant topography $\delta z=z_{0}=5 \mathrm{~nm}, \delta F / \bar{F}=0.228 \times 5 / 50$ $=0.0228$, and $F \approx \bar{F}(1+0.0228)=-54.79 \mathrm{pN}$. This corresponds to shortening the reference separation by $z_{0}$. For verification, we also compute the total force $\bar{F}$ from the reference solution with this new separation $d-z_{0}$ (with the same $\theta$ and $L$, which completely define the geometry and which correspond to a $10 \%$ smaller $R$ ), which equals $-54.49 \mathrm{pN}$, indicating the validity of the method.

In conclusion, an analytic perturbation solution to the potential between a hyperboloidal tip and a rough surface has been derived for a particular tip-surface spacing in terms of
TABLE I. Sample topography, its azimuth-averaged counterpart and the corresponding first-order corrections for the electrostatic force. Here, $z_{0}$ is the characteristic height of the topography, $x$ one of the Cartesian coordinates, $J_{0}$ the Bessel function of the first kind of order 0 , and $X$ any length parameter (as long as $X>>z_{0}$ ).

\begin{tabular}{ccc}
\hline \hline$\delta z$ & $\delta \tilde{z}$ & $\delta F / \bar{F}$ \\
\hline$z_{0}$ & $z_{0}$ & $0.228 z_{0} / a$ \\
$z_{0} \sin (2 \pi x / X)$ & 0 & 0 \\
$z_{0} \cos [2 \pi x /(2 a)]$ & $z_{0} J_{0}\left(2 \pi \sqrt{\xi^{2}-1} / 2\right)$ & $0.0189 z_{0} / a$ \\
$z_{0} \cos [2 \pi x /(3 a)]$ & $z_{0} J_{0}\left(2 \pi \sqrt{\xi^{2}-1} / 3\right)$ & $0.0474 z_{0} / a$ \\
$z_{0} \cos [2 \pi x /(4 a)]$ & $z_{0} J_{0}\left(2 \pi \sqrt{\xi^{2}-1} / 4\right)$ & $0.0741 z_{0} / a$ \\
$z_{0} \cos [2 \pi x /(5 a)]$ & $z_{0} J_{0}\left(2 \pi \sqrt{\xi^{2}-1} / 5\right)$ & $0.0963 z_{0} / a$ \\
\hline \hline
\end{tabular}

the prolate spheroidal coordinates. With this solution, the tipsurface electrostatic force can also be computed which is found to be only dependent on the azimuth-averaged topography. Sample calculations are performed for constant and sinusoidal topographies, of which only constant and cosine spatial waves (but not sine waves) contribute to the force correction. A generic topography $\delta z$ that can be modeled as a superposition of two-dimensional Fourier components can thus be analyzed within this framework, provided $\|\delta z\| \ll a$ and $\|\nabla(\delta z)\| \ll 1$. This result can be used to interpret EFM images by analyzing the horizontal force variations as the tip scans a sample.

This work was supported by the Global Climate and Energy Project at Stanford University.

${ }^{1}$ D. Vandembroucq and S. Roux, Phys. Rev. E 55, 6171 (1997).

${ }^{2}$ Z. Hurych, Solid-State Electron. 9, 967 (1966).

${ }^{3}$ A. García-Valenzuela, N. C. Bruce, and D. Kouznetsov, J. Phys. D 31, 240 (1998)

${ }^{4}$ A. García-Valenzuela, N. C. Bruce, and D. Kouznetsov, Appl. Phys. Lett. 77, 2066 (2000).

${ }^{5}$ L. Boyer, F. Houzé, A. Tonck, J.-L. Joubet, and J.-M. Georges, J. Phys. D 27, 1504 (1994).

${ }^{6}$ J. E. Stern, B. D. Terris, H. J. Mamin, and D. Rugar, Appl. Phys. Lett. 53, 2717 (1988).

${ }^{7}$ P. Girard, Nanotechnology 12, 485 (2001).

${ }^{8}$ W. R. Smythe, Static and Dynamic Electricity (McGraw-Hill, New York, 1968).

${ }^{9}$ V. J. Peridier, L.-H. Pan, and T. E. Sullivan, J. Appl. Phys. 78, 4888 (1995).

${ }^{10} \mathrm{C}$. Snow, Hypergeometric and Legendre Functions with Applications to Integral Equations of Potential Theory (National Bureau of Standards, Washington, 1952), pp. 289-300.

${ }^{11}$ R. G. van Nostrand, J. Math. Phys. (Cambridge, Mass.) 33, 276 (1954). 